\title{
ERGODIC THEOREMS FOR FOURIER TRANSFORMS OF NONCOMMUTATIVE ANALOGUES OF VECTOR MEASURES
}

\author{
KARI YLINEN
}

\begin{abstract}
Let $G$ be a locally compact group and $E$ a complex Banach space. Let $\phi: G \rightarrow E$ be a function which is the Fourier transform of a weakly compact operator $\Phi: C^{*}(G) \rightarrow E$ in the sense that $\Phi^{* *}(\omega(s))=\phi(s), s \in G$, where $\omega: G \rightarrow W^{*}(G) \subset L\left(H_{\omega}\right)$ corresponds to the universal representation of $C^{*}(G)$. It is proved that $\lim _{i} \int \phi d \mu_{i}=\Phi^{* *}\left(p_{\omega}\right)$, where $p_{\omega}$ is the projection onto the space of the common fixed points of all $\omega(s), s \in G$, and $\left(\mu_{i}\right)_{i \in I}$ is an arbitrary net in the measure algebra $M(G)$ satisfying $\sup _{i \in I}\left\|\omega\left(\mu_{i}\right)\right\|<\infty$, $\lim _{i} \mu_{i}(G)=1$, and $\lim _{i}\left\|\omega\left(\mu_{i} * \delta_{s}-\mu_{i}\right) \xi\right\|=\lim _{i}\left\|\omega\left(\mu_{i}^{*} * \delta_{s}-\mu_{i}^{*}\right) \xi\right\|=0$ for all $s \in G, \xi \in H_{\omega}$. If $E$ is a Hilbert space and $\phi$ left (resp. right) homogeneous, the second (resp. first) of the last two limit conditions may be omitted. Finally, a connection of such random fields $\phi$ to a measurability condition is established.
\end{abstract}

1. Introduction and notation. The classical mean ergodic theorem for wide sense stationary stochastic processes is a simple consequence of their spectral representation (see e.g. [7, pp. 489,$529 ; 19$, p. 10]). Since such a process $\phi$ is expressible in the form $t \mapsto U(t) \phi(0)$ for a continuous unitary representation $U$ of the time domain, the result may also be directly seen as an instance of von Neumann's mean ergodic theorem. The former approach has been generalized by Ponomarenko $[\mathbf{1 5}$, Theorem 3] for Fourier transforms of Banach space valued Borel vector measures on the dual group of a locally compact Abelian group with a certain "ergodic" sequence of functions. As exemplified by [18] (for related early work, see also [2, $\mathbf{3}, \mathbf{5}]$ ), in the noncommutative setting the study of ergodic properties of stochastic processes (i.e., random fields) has traditionally remained in the generalized time domain in keeping with the latter approach.

In this paper we switch the point of view by considering certain Banach and Hilbert space valued functions on a locally compact group as Fourier transforms of noncommutative analogues of vector measures in the sense of $[\mathbf{2 0}]$, and prove for such functions two closely related ergodic theorems. The first (Theorem 2.5) significantly generalizes [15, Theorem 3], and the second (Theorem 3.1) is shown to extend and complement [18, Theorem 2.1] on left or right homogeneous measurable random fields. The main innovation here consists in the use of the group $C^{*}$-algebra and its bidual in proving ergodic theorems for functions defined on the group.

We let $G$ denote a locally compact (not necessarily Abelian) group, $C^{*}(G)$ the group $C^{*}$-algebra of $G$, and $W^{*}(G)$ the bidual of $C^{*}(G)$ endowed with its usual $W^{*}$-algebra structure so that $W^{*}(G)$ is identified with the von Neumann algebra

Received by the editors August 14, 1985.

1980 Mathematics Subject Classification (1985 Revision). Primary 22D40, 43-00, 43A30; Secondary 60G60.

Key words and phrases. (Amenable) locally compact group, group $C^{*}$-algebra, convergence to right invariance, ergodic theorem, homogeneous random field. 
generated by the image of $C^{*}(G)$ under the universal representation $\omega: C^{*}(G) \rightarrow$ $L\left(H_{\omega}\right)$. We also let $\omega$ denote the corresponding continuous unitary representation of $G$ as well as its natural extension to $M(G)$, the convolution Banach *-algebra of bounded complex Radon measures (identified with the regular complex Borel measures on $G$ ) [6, pp. 282-283]. The convolution of $\mu, \nu \in M(G)$ is denoted by $\mu * \nu$, and $\delta_{s}$ is the Dirac measure at $s \in G$. The notation $\|\mu\|^{\prime}=\|\omega(\mu)\|$ will be used for $\mu \in M(G)$. We let $E$ denote a complex Banach space. Generalizing the Fourier (-Stieltjes) transform of a regular Borel vector measure on the dual group of a locally compact Abelian group, the Fourier transform of a weakly compact operator $\Phi: C^{*}(G) \rightarrow E$ is the function $\phi: G \rightarrow E$ defined by $\phi(s)=\Phi^{* *}(\omega(s)$ ). (Note that $\phi(s) \in E$, since the bitranspose $\Phi^{* *}$ of $\Phi$ maps $W^{*}(G)$ into $E \subset E^{* *}$.) For motivation, details, and references to background material, see [20]. In particular, [20] contains several characterizations of the vector functions arising as Fourier transforms in this sense.

An important role is played by the orthogonal projection of the Hilbert space $H_{\omega}$ onto the fixed point space $\left\{\xi \in H_{\omega} \mid \omega(s) \xi=\xi\right.$ for all $\left.s \in G\right\}$; we always denote this projection by $p_{\omega}$. Note that since $\omega(s) \in W^{*}(G)$, the projection onto each $\operatorname{ker}(1-\omega(s))$ belongs to $W^{*}(G)$, and so $p_{\omega}$, being their infimum, is also in $W^{*}(G)$.

2. The main result. At least since [2], various averaging sequences and nets to serve the needs of noncommutative ergodic theory have been studied (see e.g. [18, $\S 1]$ for one such notion and a long list of examples). Some sort of convergence to left or right invariance is the central theme and is closely tied with the amenability of $G$ [10]. The following definition is in line with this development.

2.1. Definition. The net $\left(\mu_{i}\right)_{i \in I}$ in $M(G)$ is said to be $\omega$-strongly convergent to right invariance if $\lim _{i}\left\|\omega\left(\mu_{i} * \delta_{s}-\mu_{i}\right) \xi\right\|=0$ for all $s \in G$ and $\xi \in H_{\omega}$.

2.2. REMARK. The somewhat abstract condition above has an equivalent reformulation directly in terms of $G$ :

$$
\lim _{i} \int f(t) d\left(\left(\mu_{i} * \delta_{s}-\mu_{i}\right)^{*} *\left(\mu_{i} * \delta_{s}-\mu_{i}\right)\right)(t)=0
$$

for every $s \in G$ and every continuous positive-definite function $f$ on $G$. This follows easily from the considerations in [9, pp. 193-194].

The proof of the following lemma merely modifies a well-established pattern descending from [3, p. 307] or even from [17, p. 275] (compare [5, pp. 184-185; 18, pp. 204-206; 16, pp. 56-57; 11, p. 33; 12, pp. 178-179]).

2.3. LEMMA. Let $\left(\mu_{i}\right)_{i \in I}$ be a $\|\cdot\|^{\prime}$-bounded net in $M(G)$, $\omega$-strongly convergent to right invariance and satisfying $\lim _{i} \mu_{i}(G)=1$. Then $\lim _{i}\left\|\omega\left(\mu_{i}\right) \xi-p_{\omega} \xi\right\|=0$ for all $\xi \in H_{\omega}$.

Proof. Suppose $\xi \in H_{\omega}$ and $\varepsilon>0$. From the Alaoglu-Birkhoff mean ergodic theorem as formulated e.g. in [4, p. 371] it follows that we can find $s_{1}, \ldots, s_{n} \in G$ and $\alpha_{1}, \ldots, \alpha_{n} \in[0,1]$ such that $\sum_{k=1}^{n} \alpha_{k}=1$ and for $\xi_{0}=\sum_{k=1}^{n} \alpha_{k} \omega\left(s_{k}\right) \xi$ we have $\left\|\xi_{0}-p_{\omega} \xi\right\|<\varepsilon / 2 C$, where $C>\sup _{i \in I}\left\|\mu_{i}\right\|^{\prime}$. Since

$$
\left(\omega\left(\mu_{i}\right) p_{\omega} \xi \mid \eta\right)=\int\left(\omega(s) p_{\omega} \xi \mid \eta\right) d \mu_{i}(s)=\mu_{i}(G)\left(p_{\omega} \xi \mid \eta\right)
$$


for all $\eta \in H_{\omega}$, we have $\omega\left(\mu_{i}\right) p_{\omega} \xi=\mu_{i}(G) p_{\omega} \xi$. Since $\lim _{i} \mu_{i}(G)=1$, there is thus $i_{0} \in I$ such that

$$
\begin{aligned}
& \left\|p_{\omega} \xi-\omega\left(\mu_{i}\right) \xi_{0}\right\|=\left|\mu_{i}(G)^{-1}\right|\left\|\omega\left(\mu_{i}\right) p_{\omega} \xi-\omega\left(\mu_{i}\right) \mu_{i}(G) \xi_{0}\right\| \\
& \quad \leq\left|\mu_{i}(G)^{-1}\right| C\left(\left\|p_{\omega} \xi-\xi_{0}\right\|+\left\|\xi_{0}-\mu_{i}(G) \xi_{0}\right\|\right)<\varepsilon / 2, \quad \text { when } i \geq i_{0} .
\end{aligned}
$$

Thus there is $i_{1} \in I$ such that

$$
\begin{aligned}
& \left\|p_{\omega} \xi-\omega\left(\mu_{i}\right) \xi\right\|<\frac{\varepsilon}{2}+\left\|\omega\left(\mu_{i}\right) \xi_{0}-\omega\left(\mu_{i}\right) \xi\right\| \\
& \quad=\frac{\varepsilon}{2}+\left\|\sum_{k=1}^{n} \alpha_{k}\left[\omega\left(\mu_{i} * \delta_{s_{k}}\right) \xi-\omega\left(\mu_{i}\right) \xi\right]\right\| \\
& \quad \leq \frac{\varepsilon}{2}+\sum_{k=1}^{n} \alpha_{k}\left\|\omega\left(\mu_{i} * \delta_{s_{k}}-\mu_{i}\right) \xi\right\|<\varepsilon, \quad \text { when } i \geq i_{1} .
\end{aligned}
$$

2.4. REMARK. Since $\left\|\mu_{i}\right\|^{\prime}=\left\|\mu_{i}^{*}\right\|^{\prime}$ and $\mu_{i}(G)=\overline{\mu_{i}^{*}(G)}$, it immediately follows from Lemma 2.3 that if $\left(\mu_{i}\right)_{i \in I}$ is a $\|\cdot\|^{\prime}$-bounded net with $\lim _{i} \mu_{i}(G)=1$ and such that $\left(\mu_{i}^{*}\right)_{i \in I}$ is $\omega$-strongly convergent to right invariance, then

$$
\lim _{i}\left\|\omega\left(\mu_{i}^{*}\right) \xi-p_{\omega} \xi\right\|=0
$$

for all $\xi \in H_{\omega}$. Instead of formulating a left-handed version of Definition 2.1 we prefer to work with $\left(\mu_{i}^{*}\right)_{i \in I}$. This enables us to avoid some inessential notational complications. In fact, it turns out that a natural requirement in the left-handed version would be $\lim _{i}\left\|\omega\left(\delta_{s} * \mu_{i}-\mu_{i}\right)^{*} \xi\right\|=0$.

We now assume that for the Banach space $E, \phi: G \rightarrow E$ is the Fourier transform of some weakly compact operator $\Phi: C^{*}(G) \rightarrow E$. The defining relation $\phi(s)=$ $\Phi^{* *}(\omega(s))$ has the natural extension

$$
\int \phi d \mu=\Phi^{* *}(\omega(\mu))
$$

for all $\mu \in M(G)[20$, p. 362]; the integral on the left makes sense because $\phi$ is bounded and continuous [20, pp. 360-362]. Now $\Phi^{* *}$ is continuous from $\sigma\left(W^{*}(G), C^{*}(G)^{*}\right)$ to $\sigma\left(E, E^{*}\right)$, and so

$$
\lim _{i} \int \phi d \mu_{i}=\Phi^{* *}\left(p_{\omega}\right)
$$

with respect to $\sigma\left(E, E^{*}\right)$ whenever

$$
\lim _{i} \omega\left(\mu_{i}\right)=p_{\omega}
$$

with respect to $\sigma\left(W^{*}(G), C^{*}(G)^{*}\right)$. Since the strong operator topology on $W^{*}(G)$ is finer than the weak operator topology which agrees with $\sigma\left(W^{*}(G), C^{*}(G)^{*}\right)[6$, p. 265], from Lemma 2.3 and Remark 2.4 combined with the $\sigma\left(W^{*}(G), C^{*}(G)^{*}\right)$ continuity of the involution in $W^{*}(G)$ it follows that this is the case whenever $\left(\mu_{i}\right)_{i \in I}$ is a $\|\cdot\|^{\prime}$-bounded net in $M(G)$ such that $\lim _{i} \mu_{i}(G)=1$ and $\left(\mu_{i}\right)_{i \in I}$ or $\left(\mu_{i}^{*}\right)_{i \in I}$ is $\omega$-strongly convergent to right invariance. However, in order to prove norm convergence in (2) we replace "or" with "and." 
2.5. THEOREM. Let $\left(\mu_{i}\right)_{i \in I}$ be a $\|\cdot\|^{\prime}$-bounded net in $M(G)$ with $\lim _{i} \mu_{i}(G)=$ 1. Suppose that both $\left(\mu_{i}\right)_{i \in I}$ and $\left(\mu_{i}^{*}\right)_{i \in I}$ are $\omega$-strongly convergent to right invariance. Then

$$
\lim _{i}\left\|\int \phi d \mu_{i}-\Phi^{* *}\left(p_{\omega}\right)\right\|=0 \text {. }
$$

PROOF. Lemma 2.3 combined with Remark 2.4 shows that both $\lim _{i} \omega\left(\mu_{i}\right)=p_{\omega}$ and $\lim _{i} \omega\left(\mu_{i}\right)^{*}=p_{\omega}$ with respect to the strong operator topology of $L\left(H_{\omega}\right)$. It follows that

$$
\lim _{i} \omega\left(\mu_{i}\right) \omega\left(\mu_{i}\right)^{*}=\lim _{i} \omega\left(\mu_{i}\right)^{*} \omega\left(\mu_{i}\right)=p_{\omega}^{2}=p_{\omega}
$$

with respect to the weak operator topology, and so with respect to $\sigma\left(W^{*}(G), C^{*}(G)^{*}\right)$ $[6$, p. 265]. The rest of the proof depends on a technique we also used in $[20, \mathrm{p}$. $361]$. Let $\varepsilon>0$ be given. Using Theorem II. 3 in $[\mathbf{1}$, p. 289] we find a positive linear functional $g \in C^{*}(G)^{*}$ and $\delta>0$ such that when we denote $C=\sup _{i}\left\|\omega\left(\mu_{i}\right)\right\|+1$ we have $\left|\left\langle\Phi^{*} f, u\right\rangle\right|<\varepsilon / 2 C$ for all $f \in E^{*}$ with $\|f\| \leq 1$ whenever $u \in W^{*}(G)$, $\|u\| \leq 1$, and $g\left(u^{*} u+u u^{*}\right)<\delta$. Denoting $u_{i}=\left(\omega\left(\mu_{i}\right)-p_{\omega}\right) / 2 C$ we have $\left\|u_{i}\right\| \leq 1$ and $\lim _{i} g\left(u_{i}^{*} u_{i}+u_{i} u_{i}^{*}\right)=0$ by (3) and the fact that the involution in $W^{*}(G)$ is $\sigma\left(W^{*}(G), C^{*}(G)^{*}\right)$-continuous. Thus there is $i_{0} \in I$ such that $i \geq i_{0}$ implies

$$
\left|\left\langle f, \Phi^{* *}\left(\omega\left(\mu_{i}\right)-p_{\omega}\right)\right\rangle\right|=\left|\left\langle\Phi^{*} f, 2 C u_{i}\right\rangle\right|<\varepsilon \quad \text { for all } f \in E^{*},
$$

$\|f\| \leq 1$, i.e., $\left\|\int \phi d \mu_{i}-\Phi^{* *}\left(p_{\omega}\right)\right\|<\varepsilon$ by $(1)$.

2.6. REMARKS. (a) If $\left(\nu_{i}\right)_{i \in I}$ is a $\|\cdot\|^{\prime}$-bounded net in $M(G)$, $\omega$-strongly convergent to right invariance, and with $\lim _{i} \mu_{i}(G)=1$, it is easy to verify that the net $\left(\mu_{i}\right)_{i \in I}$ with $\mu_{i}=\nu_{i}^{*} * \nu_{i}$ satisfies the hypotheses of Theorem 2.5.

(b) If $G$ is amenable, there are nets $\left(\mu_{i}\right)_{i \in I}$ with much stronger properties than used in Theorem 2.5. For example, if $\left(\nu_{i}\right)_{i \in I}$ is a net of (absolutely continuous probability) measures strongly convergent to left invariance $\left[10\right.$, p. 33], take $\mu_{i}=$ $\nu_{i} * \nu_{i}^{*}$. It is not immediately apparent if, conversely, the existence of a net as in Theorem 2.5 forces $G$ to be amenable; the net $\left(\mu_{i}\right)_{i \in I}$ is not assumed to be bounded in the total variation norm $\|\cdot\|\left(\geq\|\cdot\|^{\prime}\right)$, and we are only assuming a rather weak form of convergence to right invariance. However, $G$ must be amenable, if there is in $M(G)$ a net $\left(\mu_{i}\right)_{i \in I}$ with $C=\sup _{i \in I}\left\|\mu_{i}\right\|<\infty, \lim _{i} \mu_{i}(G)=1$, and $\lim _{i}\left\|\mu_{i} * \delta_{s}-\mu_{i}\right\|=0$ (resp. $\lim _{i}\left\|\delta_{s} * \mu_{i}-\mu_{i}\right\|=0$ ) for all $s \in G$ (so that in particular left or right ergodic nets of functions in the sense of [18] can only exist for amenable locally compact groups). To see this, it is enough to consider the latter case (of convergence to left invariance), since $\left\|\delta_{s} * \mu_{i}^{*}-\mu_{i}^{*}\right\|=\left\|\left(\mu_{i} * \delta_{s^{-1}}-\mu_{i}\right)^{*}\right\|$. Modifying an argument in [10, p. 34], regard $\left(\mu_{i}\right)_{i \in I}$ as a net in the weak* compact ball with center 0 and radius $C$ in the dual of $C(G)$, the Banach space of the bounded continuous complex functions on $G$. Passing to a subnet if necessary, we may assume that the net converges weak ${ }^{*}$ to some $\mu \in C(G)^{*}$. It is easy to verify that $\mu$ is left invariant, and $\mu \neq 0$, since $\mu(1)=1$. A standard method [13, p. 168] followed by normalization produces a left invariant mean on $C(G)$, so $G$ is amenable.

2.7. EXAMPLE. Let $G$ be commutative and $I=\mathrm{N}$ so that we have a sequence of measures in Theorem 2.5; its existence is guaranteed if $G$ is $\sigma$-compact (for a more general result and references, see $[8, p .242])$. Let $\Gamma$ denote the dual group of $G$. We identify $C^{*}(G)$ with $C_{0}(\Gamma)$ as in $[20, \S 5]$ via an isomorphism defined as the unique isometric extension of the Fourier transformation on $L^{1}(G)$ (regarded as a 
subspace of $\left.C^{*}(G)\right)$. Since $C_{0}(\Gamma)^{*}=M(\Gamma)$, the $C^{*}$-algebra $\mathcal{F}(\Gamma)$ of the bounded complex Borel functions on $\Gamma$ thus becomes identified with a ${ }^{*}$-subalgebra of $W^{*}(G)$ via $\langle\nu, f\rangle=\int_{\Gamma} f d \nu$ (see e.g. [20, p. 377]). It is not difficult to verify that $\omega\left(\mu_{i}\right)$ becomes identified with the Fourier-Stieltjes transform $\hat{\mu}_{i}$ (the techniques of $[\mathbf{2 0}$, p. 373] will work; compare also [14, p. 30]). From Lemma 2.3 we conclude (see the argument following (2)) that (using the identification $C^{*}(G)^{*}=M(\Gamma)$ )

$$
\lim _{i \rightarrow \infty} \int \hat{\mu}_{i} d \nu=\left\langle\nu, p_{\omega}\right\rangle
$$

for all $\nu \in M(\Gamma)$. Since $\sup _{i \in \mathbf{N}}\left\|\hat{\mu}_{i}\right\|_{\infty}=\sup _{i \in \mathbf{N}}\left\|\omega\left(\mu_{i}\right)\right\|<\infty$, it follows in particular that there is a function $f \in \mathcal{F}(\Gamma)$ such that $\lim _{i \rightarrow \infty} \hat{\mu}_{i}(\gamma)=f(\gamma)=\left\langle\delta_{\gamma}, p_{\omega}\right\rangle$ for all $\gamma \in \Gamma$, and from the dominated convergence theorem it follows by (4) that $\int f d \nu=\left\langle\nu, p_{\omega}\right\rangle$ for all $\nu \in M(\Gamma)$, i.e., $f$ is identified with $p_{\omega}$. Since $\omega(s) p_{\omega}=p_{\omega}$ for all $s \in G$, multiplication by the continuous characters of $\Gamma$ does not change $f$, and as these separate every $\gamma \in \Gamma \backslash\{e\}$ from the identity element $e$ of $\Gamma$, we must have $f(\gamma)=0$ for all $\gamma \in \Gamma \backslash\{e\}$. Moreover, $f(e)=\lim _{i \rightarrow \infty} \hat{\mu}_{i}(e)=\lim _{i \rightarrow \infty} \mu_{i}(G)=1$. In short, we have proved that $p_{\omega}$ is naturally identified with $\chi_{\{e\}}$, so that if $m$ is the regular Borel vector measure on $\Gamma$ corresponding to the weakly compact operator $\Phi: C^{*}(G) \rightarrow E$ as in $\left[20\right.$, p. 374], we have $\Phi^{* *}\left(p_{\omega}\right)=m(\{e\})$. In particular, Theorem 3 in [15] (after an obvious translational normalization) appears as a special case of Theorem 2.5 .

3. Homogeneous and related random fields. We conclude with a discussion of an important specialized situation. Let $H$ denote a (complex) Hilbert space with inner product $(\cdot \mid \cdot)$. A function (or "random field") $\phi: G \rightarrow H$ is called left (resp. right) homogeneous if $(\phi(s) \mid \phi(t))=(\phi(u s) \mid \phi(u t))$ (resp. $(\phi(s) \mid \phi(t))=$ $(\phi(s u) \mid \phi(t u)))$ for all $s, t, u \in G$. If such a $\phi$ is weakly continuous, it is the Fourier transform of some bounded (i.e., weakly compact, since $H$ is reflexive) linear operator $\Phi: C^{*}(G) \rightarrow H\left[20\right.$, p. 381]. In such a case the requirements on $\left(\mu_{i}\right)_{i \in I}$ in Theorem 2.5 may be somewhat relaxed.

3.1. THEOREM. Let $\phi: G \rightarrow H$ be the Fourier transform of a bounded linear operator $\Phi: C^{*}(G) \rightarrow H$. Let $\left(\mu_{i}\right)_{i \in I}$ be a $\|\cdot\|^{\prime}$-bounded net in $M(G)$ such that $\lim _{i} \mu_{i}(G)=1$. If $\left(\mu_{i}\right)_{i \in I}$ (resp. $\left.\left(\mu_{i}^{*}\right)_{i \in I}\right)$ is $\omega$-strongly convergent to right invariance and $\phi$ is left (resp. right) homogeneous, then

$$
\lim _{i}\left\|\int \phi d \mu_{i}-\Phi^{* *}\left(p_{\omega}\right)\right\|=0
$$

PrOOF. Assume first that $\left(\mu_{i}\right)_{i \in I}$ is $\omega$-strongly convergent to right invariance and $\phi$ is left homogeneous. There is a positive linear functional $f$ on $C^{*}(G)$ such that $(\Phi(x) \mid \Phi(y))=\left\langle y^{*} x, f\right\rangle$ for all $x, y \in C^{*}(G)[20$, p. 381]. Using the fact that $\Phi^{* *}: W^{*}(G) \rightarrow H$ is weak*-to-weak continuous, the involution in $W^{*}(G)$ is weak $^{*}$ continuous and multiplication separately weak ${ }^{*}$ continuous, then from the weak* density of $C^{*}(G)$ in $W^{*}(G)$ we conclude that $\left(\Phi^{* *} x \mid \Phi^{* *} y\right)=\left\langle f, y^{*} x\right\rangle$ for all 
$x, y \in W^{*}(G)$. Thus by (1) in $\S 2$ we get

$$
\begin{aligned}
\| \int & \phi d \mu_{i}-\Phi^{* *}\left(p_{\omega}\right) \|^{2} \\
= & \left(\Phi^{* *}\left(\omega\left(\mu_{i}\right)\right) \mid \Phi^{* *}\left(\omega\left(\mu_{i}\right)\right)\right)-\left(\Phi^{* *}\left(\omega\left(\mu_{i}\right)\right) \mid \Phi^{* *}\left(p_{\omega}\right)\right) \\
& -\left(\Phi^{* *}\left(p_{\omega}\right) \mid \Phi^{* *}\left(\omega\left(\mu_{i}\right)\right)\right)+\left(\Phi^{* *}\left(p_{\omega}\right) \mid \Phi^{* *}\left(p_{\omega}\right)\right) \\
= & \left\langle f, \omega\left(\mu_{i}\right)^{*} \omega\left(\mu_{i}\right)\right\rangle-\left\langle f, p_{\omega} \omega\left(\mu_{i}\right)\right\rangle-\left\langle f, \omega\left(\mu_{i}\right)^{*} p_{\omega}\right\rangle+\left\langle f, p_{\omega}^{2}\right\rangle \rightarrow 0,
\end{aligned}
$$

because by Lemma $2.3 \omega\left(\mu_{i}\right) \rightarrow p_{\omega}$ strongly, and there is $\xi \in H_{\omega}$ such that $\langle f, x\rangle=$ $(x \xi \mid \xi)$ for all $x \in W^{*}(G)[6$, p. 265]. This proves the theorem in the first case. If $\left(\mu_{i}\right)_{i \in I}$ is $\omega$-strongly convergent to right invariance, then $\omega\left(\mu_{i}^{*}\right) \rightarrow p_{\omega}$ strongly (Remark 2.4), and if $\phi$ is right homogeneous, then $(\Phi(x) \mid \Phi(y))=\left\langle f, x y^{*}\right\rangle$ for some positive linear functional $f$ on $C^{*}(G)[20$, p. 381]. Taking into account these changes, the same argument as above may be used.

3.2. REMARK. If $\phi$ is as in [21, Lemma 2] and $\left(\mu_{i}\right)_{i \in I}$ is as in Theorem 2.5 (so that both $\left(\mu_{i}\right)_{i \in I}$ and $\left(\mu_{i}^{*}\right)_{i \in I}$ are $\omega$-strongly convergent to right invariance), then the conclusion of the above theorem holds, and essentially the same proof (subsuming the two cases) works. Combining this observation with the dilation theorem of [21], one would get an alternative proof of Theorem 2.5 in the case of a Hilbert space.

Theorem 3.1 complements (by characterizing the limit $\lim _{i} \int \phi d \mu_{i}$ ) and generalizes [18, Theorem 2.1] except that [18] only assumes a measurability condition instead of continuity which is inherent in our approach. We are still going to prove a result which shows among other things that in fact the measurable case studied in [18] can be reduced to ours.

We take for granted the integration theory on locally compact Hausdorff spaces and groups as expounded in $[\mathbf{1 3}]$. We let $\lambda$ be a fixed left Haar measure of $G$, we denote by $\mathcal{M}$ the $\sigma$-algebra of $\lambda$-measurable sets, and we use the notation $d s$ in integrating with respect to $\lambda$. A function $\phi: G \rightarrow H$ is said to be weakly measurable if for all $\xi \in H$ the function $s \mapsto(\phi(s) \mid \xi)$ is $\lambda$-measurable; it is enough to require that for each compact set $K \subset G$ the restriction $(\phi(\cdot) \mid \xi) \mid K$ is $\mathcal{M}_{K}$-measurable, where $\mathcal{M}_{K}=\{A \cap K \mid A \in \mathcal{M}\}$. For example, if $(\Omega, \mathcal{A}, P)$ is a probability space, and $F: G \times \Omega \rightarrow \mathbf{C}$ is such that $F(s, \cdot) \in L^{2}=L^{2}(\Omega, \mathcal{A}, P)$ for all $s \in G$, and that for each compact $K \subset G F \mid K \times \Omega$ is measurable with respect to the product $\sigma$-algebra $\mathcal{M}_{K} \times \mathcal{A}$ and $\sup _{s \in K} \int_{\Omega}|F(s, x)|^{2} d P(x)<\infty$, then an easy application of Fubini's theorem shows that the function $\phi: G \rightarrow L^{2}$, where $\phi(s)=F(s, \cdot)$, is weakly measurable.

As usual, we regard $L^{1}(G)=L^{1}(G, \lambda)$ as embedded in $M(G)$; the formulas for the induced involution and convolution (see $[6$, p. 282; 13, p. 291]) will only be used for elements of $\mathcal{K}(G)$, the space of continuous complex functions with compact support. With a trifling deviation from [13, p. 141], a complex function is said to be in $L^{\infty}(G)$ if it is $\lambda$-measurable and in modulus less than some finite constant locally almost everywhere (l.a.e.); two such functions are identified if they agree 1.a.e. The integrals of the vector functions below are easily seen to exist in the weak sense. (We only treat the case of left homogeneity, but the right-hand version is essentially similar.) 
3.3. Proposition. Let $\phi: G \rightarrow H$ be bounded and weakly measurable, and let $\rho$ be a complex function on $G$ such that for locally almost every $t \in G$ we have $(\phi(s) \mid \phi(t))=\rho\left(t^{-1} s\right)$ for locally almost every $s \in G$. Then

(a) $\rho \in L^{\infty}(G)$,

(b)

$$
\begin{aligned}
\int\left(g^{*} * f\right)(s) \rho(s) d s & =\iint f(s) \overline{g(t)}(\phi(s) \mid \phi(t)) d s d t \\
& =\left(\int f(s) \phi(s) d s \mid \int g(t) \phi(t) d t\right)
\end{aligned}
$$

for all $f, g \in \mathcal{K}(G)$, and

(c) there is a bounded linear map $\Psi: C^{*}(G) \rightarrow H$ whose Fourier transform $\psi: G \rightarrow H$ is left homogeneous and satisfies

$$
\int f(s) \phi(s) d s=\int f(s) \psi(s) d s \quad \text { for all } f \in L^{1}(G) .
$$

Proof. (a) For a suitable $t_{0} \in G, \rho(s)=\rho\left(t_{0}^{-1}\left(t_{0} s\right)\right)=\left(\phi\left(t_{0} s\right) \mid \phi\left(t_{0}\right)\right)$ for locally almost every $s \in G$. Thus $\rho \in L^{\infty}$, since the function $s \mapsto\left(\phi\left(t_{0} s\right) \mid \phi\left(t_{0}\right)\right)$ is in $L^{\infty}(G)$.

(b) The left-hand side equals

$$
\begin{aligned}
\iint & \Delta\left(t^{-1}\right) \overline{g\left(t^{-1}\right)} f\left(t^{-1} s\right) \rho(s) d t d s \\
& =\iint \overline{g(t)} f(t s) \rho(s) d t d s=\iint \overline{g(t)} f(t s) \rho(s) d s d t \\
& =\iint \overline{g(t)} f(s) \rho\left(t^{-1} s\right) d s d t=\iint \overline{g(t)} f(s)(\phi(s) \mid \phi(t)) d s d t \\
& =\left(\int f(s) \phi(s) d s \mid \int g(t) \phi(t) d t\right) .
\end{aligned}
$$

(c) Taking $f=g$ and noting that $K(G)$ is dense in $L^{1}(G)$ we see from (b) that the continuous linear functional on $L^{1}(G)$ defined by $\rho$ is positive, and so $\rho$ is l.a.e. equal to a continuous positive-definite function $\rho_{0}[6$, p. 288]. Let $h$ be the corresponding positive linear functional on $C^{*}(G)$ (denoted by $T \rho$ in $[20$, p. 359]). Let $\tau: L^{1}(G) \rightarrow C^{*}(G)$ be the canonical injection, and define $\Psi_{0}: \tau(\mathcal{K}(G)) \rightarrow H$ by $\Psi_{0}(\tau(f))=\int f(s) \phi(s) d s$. From (b) it follows that

$$
\left(\Psi_{0}(\tau(f)) \mid \Psi_{0}(\tau(g))\right)=\int\left(g^{*} * f\right)(s) \rho_{0}(s) d s=\left\langle\tau\left(g^{*}\right) \tau(f), h\right\rangle
$$

for all $f, g \in \mathcal{K}(G)$. Taking $f=g$ we see that $\left\|\Psi_{0}(\tau(f))\right\|^{2} \leq\|h\|\|\tau(f)\|^{2}$, and since $\tau(\mathcal{K}(G))$ is dense in $C^{*}(G), \Psi_{0}$ can be extended to a bounded linear operator $\Psi: C^{*}(G) \rightarrow H$. From (6) it follows that $(\Psi x \mid \Psi y)=\left\langle y^{*} x, h\right\rangle$ for all $x, y \in C^{*}(G)$, and an argument used in the proof of Theorem 3.1 shows that $\left(\Psi^{* *} x \mid \Psi^{* *} y\right)=$ $\left\langle h, y^{*} x\right\rangle$ for all $x, y \in W^{*}(G)$; in particular $(\psi(s) \mid \psi(t))=\left\langle h, \omega(t)^{*} \omega(s)\right\rangle=\rho_{0}\left(t^{-1} s\right)$ for all $s, t \in G$, so $\psi$ is left homogeneous. The equation (5) holds by construction and (1) in $\S 2$ for all $f \in \mathcal{K}(G)$; as $\mathcal{K}(G)$ is dense in $L^{1}(G)$, the general case follows from this. 


\section{REFERENCES}

1. C. A. Akemann, The dual space of an operator algebra, Trans. Amer. Math. Soc. 126 (1967), 286-302.

2. L. Alaoglu and G. Birkhoff, General ergodic theorems, Proc. Nat. Acad. Sci. U.S.A. 25 (1939), 628-630.

3. , General ergodic theorems, Ann. of Math. 41 (1940), 293-309.

4. O. Bratteli and D. W. Robinson, Operator algebras and quantum statistical mechanics I: $C^{*}$ - and $W^{*}$-algebras, symmetry groups, decomposition of states, Texts and Monographs in Physics, Springer-Verlag, New York, Heidelberg and Berlin, 1979.

5. A. P. Calderon, A general ergodic theorem, Ann. of Math. 58 (1953), 182-191.

6. J. Dixmier, $C^{*}$-algebras, North-Holland Mathematical Library, Vol. 15, North-Holland, Amsterdam, New York and Oxford, 1977.

7. J. L. Doob, Stochastic processes, Wiley Publications in Statistics, Wiley, New York; Chapman \& Hall, London, 1953.

8. W. R. Emerson, Large symmetric sets in amenable groups and the individual ergodic theorem, Amer. J. Math. 96 (1974), 242-247.

9. P. Eymard, L'algèbre de Fourier d'un groupe localement compact, Bull. Soc. Math. France 92 (1964), 181-236.

10. F. P. Greenleaf, Invariant means on topological groups and their applications, Van Nostrand Math. Studies, No. 16, Van Nostrand Reinhold, New York, Toronto and London, 1969.

11. _ Ergodic theorems and the construction of summing sequences in amenable locally compact groups, Comm. Pure Appl. Math. 26 (1973), 29-46.

12. A. Guichardet, Systèmes dynamiques non commutatifs, Astérisque 13-14, Soc. Math. France, Paris, 1974.

13. E. Hewitt and K. A. Ross, Abstract harmonic analysis. Vol. I: Structure of topological groups. Integration theory, group representations, Grundlehren Math. Wiss. B. 115, Academic Press, New York; Springer-Verlag, Berlin, 1963.

14. O. Martikainen, Convolutions of noncommutative analogues of vector measures with applications to operator ideals, Ann. Acad. Sci. Fenn. Ser. AI Math. Dissertationes 18 (1978), $1-65$.

15. A. I. Ponomarenko, On the mean value of a positive definite operator-valued function on a group, Teor. Verojatnost. i Mat. Statist. 1 (1970), 159-165; English transl., Theor. Probab. Math. Statist. 1 (1974), 155-161.

16. P. F. Renaud, General ergodic theorems for locally compact groups, Amer. J. Math. 93 (1971), 52-64.

17. F. Riesz, Some mean ergodic theorems, J. London Math. Soc. 13 (1938), 274-278.

18. A. A. Tempel'man [A. Tempelmanas], Ergodic theorems for homogeneous generalized stochastic fields and homogeneous stochastic fields on groups, Litovsk. Mat. Sb. 2 (1962), 195-213. (Russian)

19. K. Urbanik, Lectures on prediction theory, Lecture Notes in Math., vol. 44, Springer-Verlag, Berlin, Heidelberg and New York, 1967.

20. K. Ylinen, Fourier transforms of noncommutative analogues of vector measures and bimeasures with applications to stochastic processes, Ann. Acad. Sci. Fenn. Ser. A I 1 (1975), 355-385.

21. _ Dilations of $V$-bounded stochastic processes indexed by a locally compact group, Proc. Amer. Math. Soc. 90 (1984), 378-380.

DePARTMENT OF MATHEMATICS, UNIVERSity OF TURKU, SF-20500 TURKU 50, FIN- 